\title{
HUmanoid Robotic Leg via pneumatic muscle actuators: implementation and control
}

\author{
George Andrikopoulos $(\mathbb{D} \cdot$ George Nikolakopoulos
}

Received: 12 December 2016/ Accepted: 7 August 2017

(C) The Author(s) 2017. This article is an open access publication

\begin{abstract}
In this article, a HUmanoid Robotic Leg (HURL) via the utilization of pneumatic muscle actuators (PMAs) is presented. PMAs are a pneumatic form of actuation possessing crucial attributes for the implementation of a design that mimics the motion characteristics of a human ankle. HURL acts as a feasibility study in the conceptual goal of developing a 10 degree-of-freedom (DoF) lower-limb humanoid for compliance and postural control, while serving as a knowledge basis for its future alternative use in prosthetic robotics. HURL's design properties are described in detail, while its 2-DoF motion capabilities (dorsiflexion-plantar flexion, eversion-inversion) are experimentally evaluated via an advanced nonlinear PID-based control algorithm.
\end{abstract}

Keywords Pneumatic muscle actuators - Robotic leg $\cdot$ Humanoid systems $\cdot$ Nonlinear PID control

Research supported by the Swedish Research Council (Vetenskapsrådet).

G. Andrikopoulos $(\bowtie) \cdot$ G. Nikolakopoulos

Control Engineering Group, Luleå University of

Technology, 97187 Luleå, Sweden

e-mail: geoand@ltu.se

G. Nikolakopoulos

e-mail: geonik@1tu.se

\section{Introduction}

During the past few years, there has been a large research attempt in improving the design of humanoid biped robots and produce setups with motion capabilities inspired by the smoothness, accuracy and compliance that characterize the human lower-limb motion [1-3]. To that goal, the use of inherently compliant materials in structures and actuators, characterized by flexibility and biomimetic attributes, has been an increasing interest of researchers and manufacturers.

A characteristic example of biomimetic actuation has been the Pneumatic Muscle Actuator (PMA), which is characterized by a decrease in the actuating length when pressurized, while possessing similar properties with those of the organic muscle, combined with several advantages as the ability to provide high power outputs, with relatively light weights and inherent compliance [4]. These characteristics are turning the PMA into a promising actuator choice in anthropometric technologies, while a variety of such applications have already been appeared in the literature [5]. Moreover, PMA meets the need for safety, simplicity and lightness that human-robot interaction requires and justifies its expanding utilization in medical and biorobotic applications.

A small number of human-inspired PMA-actuated leg setups have been proposed in the past fifteen years, with most related research addressing the structural 
design, modeling and control problems for undertaking motions mainly in the sagittal plane [6-16]. Specifically, [6] presented a robotic leg design with the ability to perform hip and knee 1-DOF motions, with antagonistic pairs of PMAs connected through tendons on the target joints, while the ankle joint remained passive. A similar design strategy was followed in the biped humanoid presented in [7], which incorporated pleated-type PMAs in antagonistic formations to replicate the sagittal motions of the hip, knee and ankle joints. In [8] a below-knee prosthesis for the undertaking of plantar flexion ankle movements was presented, with the goal of facilitating gait patterns, where 3 pleated-type PMAs were connected via ball joints onto the metallic structure in an asymmetrical antagonistic formation.

Similarly to the techniques used in [6] and [7], researchers in [9] presented a biped prototype with the addition of bi-articular muscles to simultaneously actuate each neighbor joints ankle-knee and knee-hip. The same design techniques were also used in later efforts [9-11] for developing lower-limb humanoids for postural analysis and control but also restricted to the sagittal motion plane. Different design approaches were incorporated in the foot design for analyzing running capabilities in [12], while alternative placement for the PMA pairs in the hip-knee formations has been considered in $[13,14]$. The most recent efforts included the development and control of a robotic knee-ankle setup, which enabled ankle movements in both sagittal and planes by incorporating PMAs in a synergistic fashion $[15,16]$. The utilized technique resembled the muscle formations in the human leg, while each PMA was connected to the metallic structure via tendon-inspired wires.

In this article, an alternative mechanical solution to the aforementioned approaches is considered, where the conceptual design of a novel HUmanoid Robotic Leg (HURL) is presented in detail. The proposed design utilizes four PMAs for reproducing the two Degree of Freedom (DoF) movement capabilities of the human ankle: (a) dorsiflexion/plantar flexion, and (b) inversion/eversion [17].

The main contribution of this work stems from the design of a humanoid leg via the utilization of PMA technology. The proposed structure concerns the conceptualization of a biomimetic design for mechanically decoupled actuation of both DOFs of an ankleinspired joint. To this purpose, the synergistic nature of the human lower leg muscles has been simplified to two antagonistic pairs of PMAs in cross-formation, thus providing the structural ability of independent control of motions in the sagittal and frontal plane. The proposed approach utilizes a 3D-print-friendly design, which allows for fast, low-cost prototyping and easy customization of its sub-parts to given dimension requirements. Such a design facilitates the adjusting of important properties like its weight and Center-ofMass (CoM), the customized incorporation of sensors and electronics in its interior design, or the adjustment of its overall durability via the incorporation of tougher materials in the bone-inspired structure (e.g. carbon-fiber rods as a "bone-marrow").

In addition, this article includes the feasibility study performed through the development and experimental evaluation of a working prototype. For the evaluation of its structural stability and mechanical properties in replicating smooth and accurate motion patterns, a model-free control scheme based on Advanced Nonlinear PID (ANPID) is implemented and tested. The reasoning behind the selection of the ANPID as the base controller for the evaluation of the robotic leg resides in its capability of providing an advanced, flexible and adjustable control performance for a custom application, without requiring the knowledge of the setup's model [18].

For the evaluation of the decoupled mechanical nature of the leg in performing its ankle's 2 DOF motion patterns, the control scheme incorporates one ANPID structure per motion. This feature significantly simplifies the tuning process for each controller, given that the positioning of the PMAs on the structure in a cross-formation minimizes the effect between movements in the sagittal and frontal planes, while such effects are robustly compensated by each ANPID controller. Finally, the selected control structure is compared to conventional PID algorithms, which have been traditionally utilized in PMA-related applications [18], in an effort to further highlight the merits of the proposed model-free control structure in producing smooth and accurate movements with performance adjustable to the setup's nonlinear properties.

The proposed robotic appliance is considered as the initial conceptual point and mandatory infrastructure for enabling future interactive applications on lowerlimb balance research, while its experimental evaluation acts as the feasibility study for the future development of a lower-limb balancing humanoid 
robot. The proposed design is evaluated as a basic motion problem, however, the conceptual design from an engineering point of view and without losing generalization, can form the basis for its expansion in developing a compliant, safe and motion accurate lower-limb humanoid, while possessing the necessary biomimetic attributes for its alternative use as a prosthetic leg appliance for the undertaking of the human ankle movements.

The rest of the article is structured as follows. In Sect. 2, the HURL design is overviewed through the conceptual goal of developing a lower-limb balancing humanoid. In Sect. 3, the design specifics of the HURL as well as its motion capabilities are presented in detail. Section 4 presents the synthesized control algorithm for the undertaking of the HURL's motion. Section 5 provides an overview of the various components utilized in the implementation of the HURL prototype, while in Sect. 6, open and closed loop experimental results for the evaluation of the HURL's 2-DoF motion capabilities are presented, with the closed loop evaluation performed via the utilization of an advanced nonlinear PID-based control algorithm. Finally, concluding remarks and comments regarding future work are provided in Sects. 7 and 8, respectively.

\section{Towards the development of a lower-limb Balancing Humanoid Robot}

The ultimate goal of the presented HURL design will be development of a lower-limb humanoid robot for future research in balance and postural control. The conceptual design of the Balancing Humanoid Robot (BAHRT) is depicted in Fig. 1, whereas its design specifics are displayed in Fig. 2a. The biologicallyinspired concept is formed on the basis of the endoskeleton dimensions of an adult human, while following a muscle-tendon-based approach for the generation of the robot's movements. This design strategy leads to a reduced-DoF alternative of the following movements: (a) ankle (dorsiflexion/plantar flexion, inversion/eversion), (b) knee (flexion/extension), and (c) hip (flexion/extension, abduction/adduction). Considering the biped design, this concept leads to a total of $10-$ DoFs.

When considering the use of PMAs for the actuation of a biomimetic design, the available contraction and its direct connection to the produced contractile
Fig. 1 Conceptual overview of the Balancing Humanoid Robot (BAHRT)

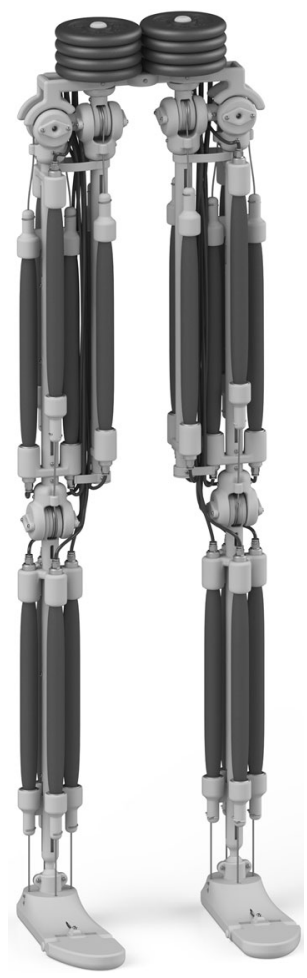

force play a major role on the joint design strategy. It has to be noted that the PMA technology provides a maximum of $25-30 \%$ permissible stroke, which when compared to the $50-70 \%$ of the biological muscle [14], creates challenges in producing similar motion ranges for given joint dimensions. This challenge is also intensified by the fact that the forces exerted on the PMAs during movement have a direct impact on its permissible contraction [4].

Taking all the above properties into consideration, and in order to produce a humanoid robot following the dimensions of an adult human, the design approach of BAHRT follows a trade-off between joint angle and torque by incorporating a biomimetic muscle-tendon strategy. In this case, the number and placement of the PMAs for the enablement of the humanoid's DOFs is properly selected so as to simplify the complexity of the various movement scenarios by taking advantage of an antagonistic motion strategy, where PMAs are working antagonistically between pairs [19]. The antagonistic PMA pairs utilized in the proposed structure are depicted in Fig. 2b, accompanied by color-matched information to the respective movements, where $\theta$ denotes the angular motion of the $i$-th joint for $i=1, \ldots, 10$. Note that joints $i=1, \ldots, 5$ and 
$i=6, \ldots, 10$ correspond to the PMA pairs used in the two symmetrical leg mechanisms.

In addition, the design involves the use of bearingbased revolute joints for the knee and hip movements, except for the motion of the ankle, where a spherical joint is appropriately attached into the ankle-inspired formation. All wire tendons are properly attached to the PMAs' end cap parts and to the respective point of rotation, where appropriate inlets on the endoskeleton structure are utilized to guide the tendons and ensure smoothness and efficiency.

In achieving the more demanding hip's 2-DoFs (flexion/extension, abduction/adduction) the concept involves the use of mechanically independent rotation points via appropriately designed endoskeletal parts, as shown in Fig. 2. This strategy, along with the use of Bowden cable mechanisms for the generation of the hip and knee joint movements, enables the PMAs involved to be placed around the Femur bone area, rather than the space around the back and abdomen, thus providing a novel design alternative. A basic advantage of this strategy is the mechanical decoupling of the hip's abduction/adduction, since they can now be performed in an independent way, which could potentially simplify the modeling and control problems.

\section{Conceptual design of the HURL}

\subsection{Design specifics}

The proposed concept, which is presented in Fig. 3 in assembled and exploded view, utilizes a 3D-printfriendly structure that allows for fast and low-cost prototyping, while supporting the easy customization of its sub-parts to given dimension and weight requirements. Such a design supports the adjusting of important properties like its weight and Center-ofMass (CoM), the future incorporation of sensors and electronics in its interior design, or the adjustment of its overall durability via the incorporation of tougher materials in the bone-inspired structure (e.g. carbonfiber rods as a "bone-marrow").

As presented in Fig. 3, mounting, protective and connection caps are assembled on a Tibia/Fibula
Fig. 2 Conceptual overview of the Balancing Humanoid Robot (BAHRT): a design specifics, b colormatched PMA pairs to the respective generated movements

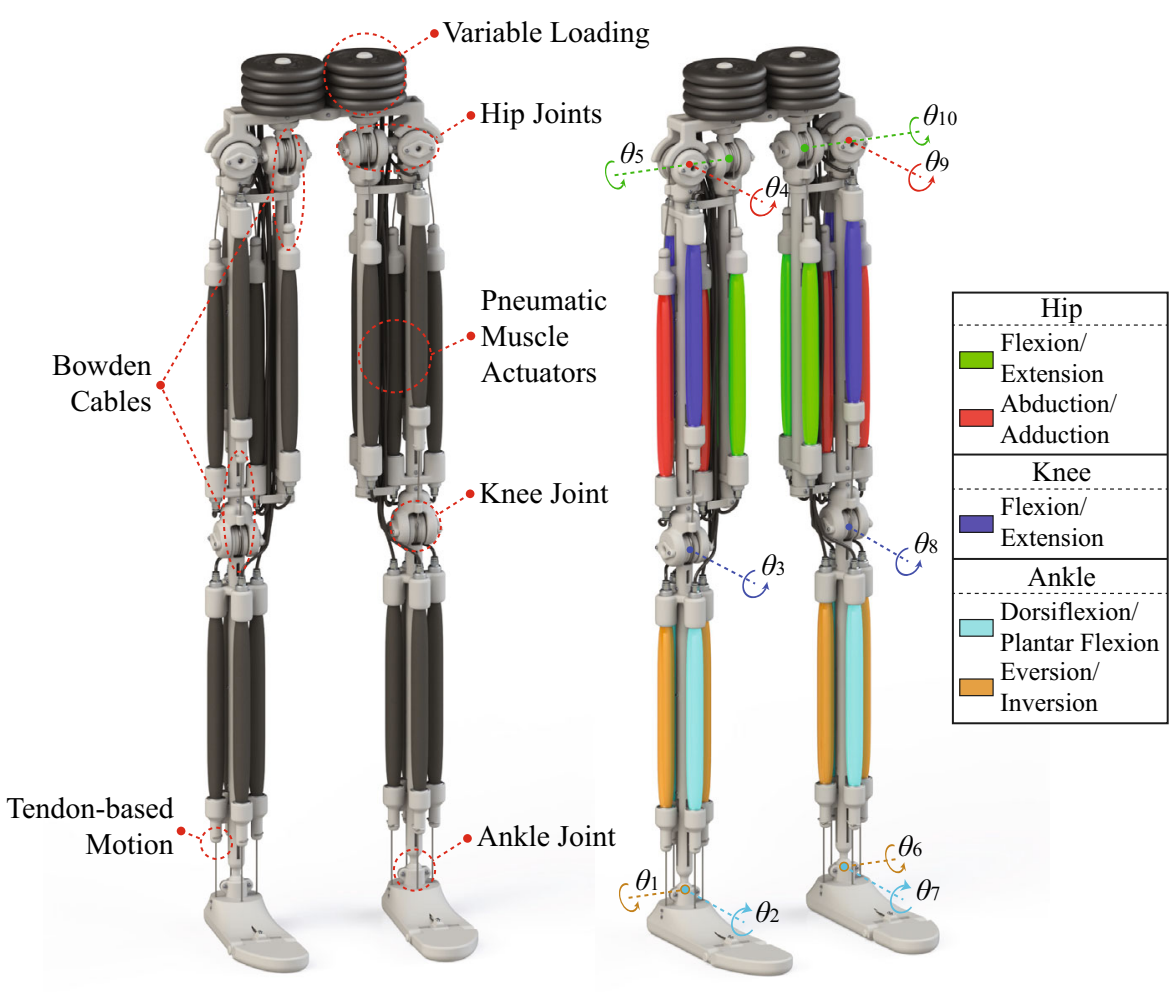

(a)

(b) 


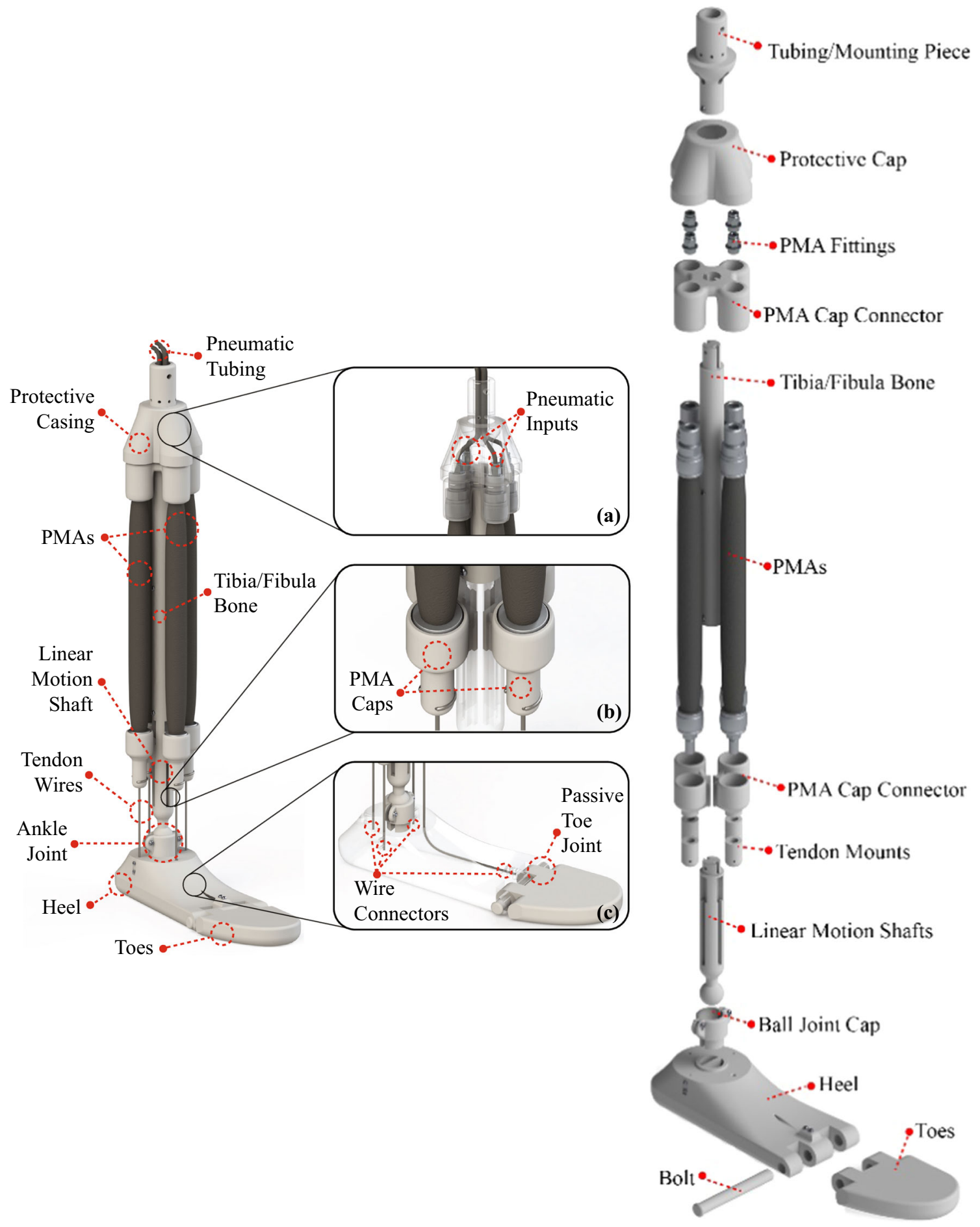

Fig. 3 Design specifics of the HURL in assembled (left) and exploded view (right)

inspired rod, which facilitates the linear motion of the PMAs. The number and placement of the PMAs for the actuation of the HURL appliance is properly chosen in order to simplify the complexity of the ankle movement scenarios (dorsiflexion/plantar flexion and inversion/eversion). The proposed design utilizes two 
antagonistic PMA pairs placed in cross-formation around the Tibia/Fibula-inspired bone and with the intersecting plane of each pair being parallel to the target plane of motion.

The motion transfer mechanism utilizes tendon wires that connect the PMAs via caps to the HURL's heel-inspired formation via appropriately placed connection points (Fig. 3c). The caps involve a choke mechanism for the placement and easy adjustment of the tendon wires during operation, while four linear motion shafts are incorporated in the Tibia/Fibula bone design for the connection with the PMA caps, which operate as prismatic joints in order to enforce the linear motion of the PMAs and compensate for any non-axial forces exerted during complex motion scenarios (Fig. 3b).

It has to be noted that the wire connection points on the heel are distributed along the spherical joint that acts as a simplified ankle-inspired mechanism, except for the front tendon wire that is responsible for the flexion movements, which is driven through the foot's bridge area and is finally attached to the connection point resembling the mound of a toe-inspired formation (Fig. 3). This particular strategy is identified as most suitable for the better distribution of forces during operation, which is crucial due to the asymmetry of the foot along its longitudinal axis, while mimicking the placement of the extensor tendons in the human foot [20], i.e. the main tendons responsible for the dorsiflexion and inversion movements. Finally, the toe-inspired formation is connected to the main foot section via a passive joint, which incorporates a torsion spring in order to restrict unwanted toe movements during the HURL's operation.

This design aspect provides the significant advantage of mechanically decoupling the HURL's 2 DoFs, as each pair of PMAs is structurally responsible for the generation of one respective movement. The design differs from the synergy-based human muscle operation, where muscle groups are involved in the generation of more than one movements of the human foot [20]. A visual representation of the biomimetic assignment of the PMAs and wire tendons to their respective main biological counterparts is presented in Fig. 4. As presented in the next Subsection, this biomimetic simplification leads to a motion strategy defined by one manipulated variable per PMA pair, thus simplifying the motion control problem.

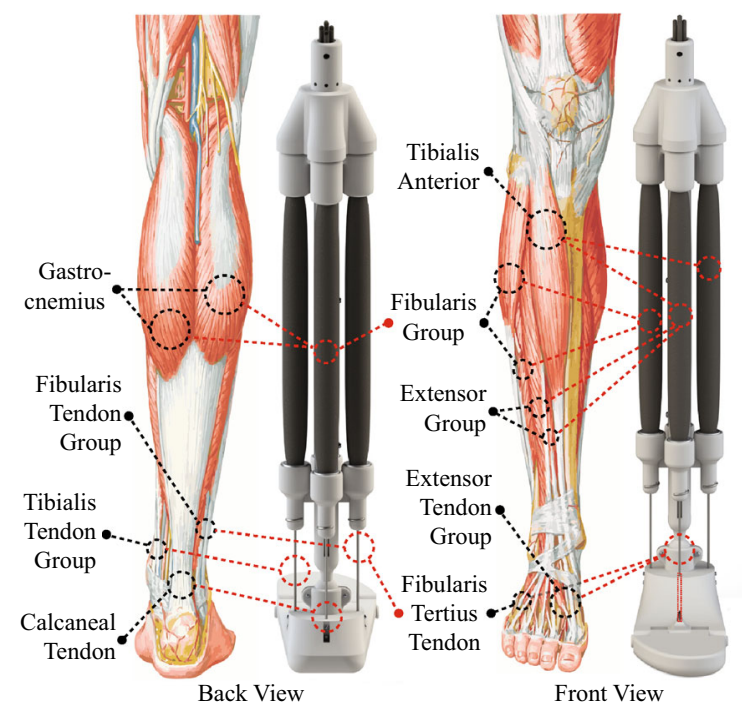

Fig. 4 Biomimetic assignment of the HURL's PMAs and tendons to their respective main biological counterparts [20]

\subsection{Motion strategy}

The selected movement strategy for the replication of the ankle's 2-DoF motion capabilities is graphically represented in Fig. 5. Green color highlights the PMAs in idle (initial) contracted state, blue color highlights the inflated PMAs, while the deflated PMAs are highlighted with red color.

In order to take advantage of the full PMA stroke and thus, ensure the maximum provided range of motion for every DoF, the PMAs are initially inflated at a $P_{0}$ pressure which corresponds to the half of their maximum permissible stroke, before being connected to their respective tendons. With that in mind, the utilized antagonistic movement strategy is formulated as:

$P_{i, j}=P_{i, 0} \pm \Delta P_{i}$

for $\left\{\begin{array}{c}i=1 \text { and } j=1,2 \text { with }\left\{\begin{array}{c}\Delta P_{1}>0 \Rightarrow \text { Dorsiflexion (Fig . 5b) } \\ \Delta P_{1}<0 \Rightarrow \text { Plantar Flexion (Fig. 5c) }\end{array}\right. \\ i=2 \text { and } j=1,2 \text { with }\left\{\begin{array}{l}\Delta P_{2}>0 \Rightarrow \text { Eversion (Fig. 5d) } \\ \Delta P_{2}<0 \Rightarrow \text { Inversion (Fig. 5e) }\end{array}\right.\end{array}\right.$

where $P_{i, j}$ defines the pressure values utilized in every antagonistic pair of PMAs and identified as $j=1,2$, for the undertaking of the $i=1,2$ DoF movements of dorsiflexion/plantar flexion and inversion/eversion respectively, while $P_{i, 0}$ denotes the initial pressure of the $i$-th PMA pair. This strategy has the advantage of 


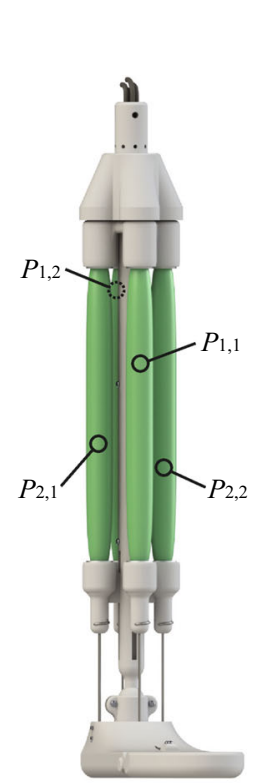

(a)

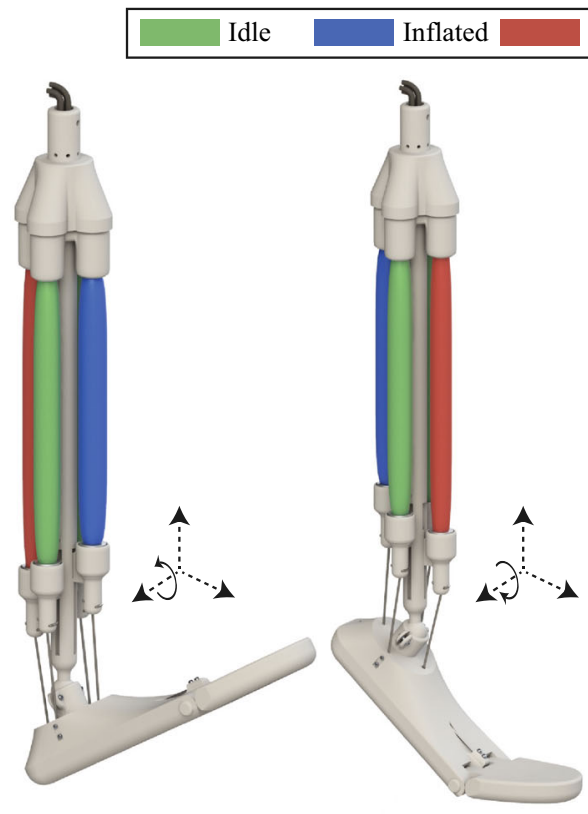

(b)

(c)

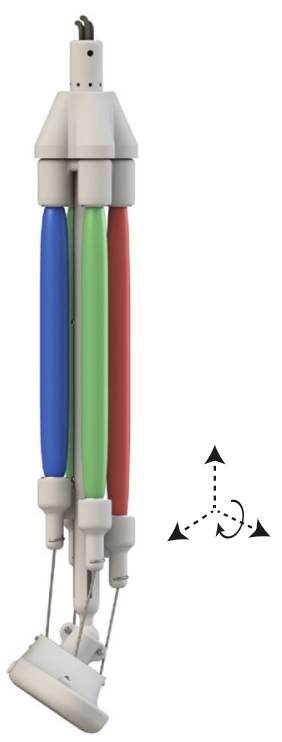

(d)

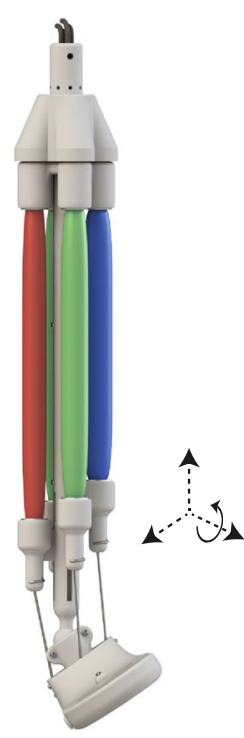

(e)

Fig. 5 Graphical representation of the HURL movement strategy during: a idle, b dorsiflexion, c plantar flexion, d eversion and e inversion

utilizing one manipulated pressure variable $\Delta P_{\mathrm{i}}$ per antagonistic PMA pair and depending on its sign leads to the activation of the target motion pattern.

\section{Motion control}

In the concept of evaluating the HURL's operation, the ANPID algorithm has been considered as a suitable choice for undertaking the motion control problem, due to its increased flexibility and adaptability, as well as its experimentally verified capability in producing smooth, fast and accurate PMA responses [18]. The purpose of the ANPID-based control scheme presented in Fig. 6 is not to provide novel knowledge on PMA control theory rather than to test and evaluate its performance on the proposed exoskeletal appliance. The development and evaluation of more complex control structures for torque and compliance control is part of future work.

The movement strategy described in (1) significantly simplifies the motion control problem, since every scenario involves only one control variable i.e. the manipulated pressure $\Delta P_{i}$, which is transferred via the same strategy to the PMAs of the HURL. For this reason, the HURL's motion control problem was addressed via the use of a double ANPID-based control structure, which is presented in detail in Fig. 5, under the assumption of a sampling process with sampling time $T_{s} \varepsilon \mathbb{R}^{+}$, while at sample $n \varepsilon \mathbb{Z}^{+}$.

The ANPID algorithm, when compared to the standard PID structure [21], features additional tuning modes for its basic control parameters, i.e. the proportional gain $K_{P}$, the reset time $T_{I}$ in minutes and the rate time $T_{D}$ in minutes, which synthesize the following modified error signals $e_{P}, e_{I}$ and $e_{D}$ for the proportional, integral and derivative control action terms, respectively:

$$
\begin{aligned}
e_{P}(n) & =\frac{\left[f x_{\mathrm{ref}}(n)-x(n)\right]}{x_{\mathrm{ref}, \text { range }}}\left[g x_{\mathrm{ref}, \text { range }}+(1-g)\left|f x_{\mathrm{ref}}(n)-x(n)\right|\right], \\
e_{I}(n) & =\frac{\left[x_{\mathrm{ref}}(n)-x(n)\right]}{x_{\mathrm{ref}, \text { range }}}\left[g x_{\mathrm{ref}, \text { range }}+(1-g)\left|x_{\mathrm{ref}}(n)-x(n)\right|\right], \\
e_{D}(n) & =\frac{\left[q x_{\mathrm{ref}}(n)-x(n)\right]}{x_{\mathrm{ref}, \text { range }}}\left[g x_{\mathrm{ref}, \text { range }}+(1-g)\left|q x_{\mathrm{ref}}(n)-x(n)\right|\right]
\end{aligned}
$$

where $x_{\text {ref, range }}$ denotes the range of the reference output $x$. The mode selector $f \varepsilon[0,1]$ is utilized as a relative trade-off between noise rejection and set-point tracking; $f=1$ results in error-affected action and control emphasis on tracking the reference signal, while $f=0$ results in measurement-affected action 


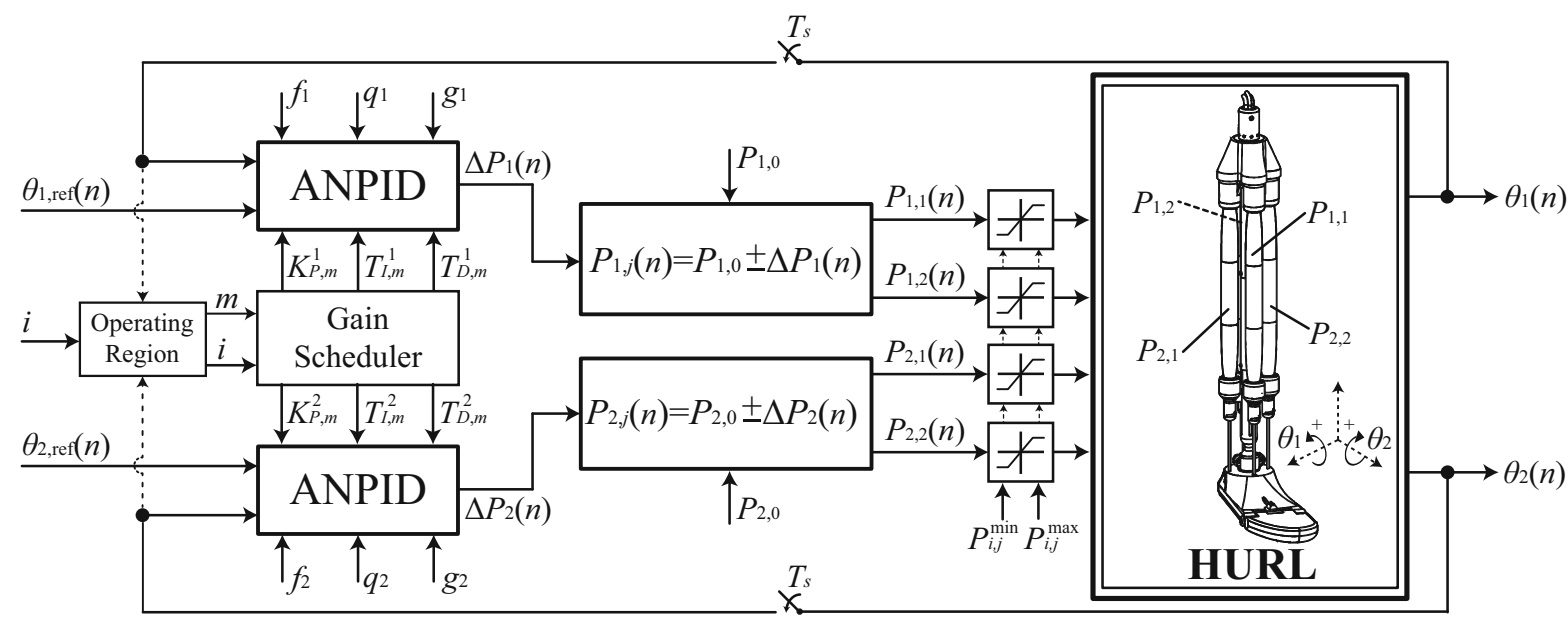

Fig. 6 The ANPID-based control scheme for the 2-DoF motion control of the HURL

and emphasis on disturbance cancellation. The mode selector $q \varepsilon[0,1]$ accounts for differentiation on error ( $q=1$ ) or measurement $(q=0)$. When $q$ is posed on measurement, it reduces the derivative kick effect and results in reverse-acting control operation [21].

Furthermore, in order to achieve an adjustable decrease of $K_{p}$ gain at low error magnitudes and the corresponding gradual increase at large deviations from the set-point, the linearity factor $g \varepsilon[0,1]$, is properly introduced in (2) to account for the increase in $K_{p}$, with respect to the error's magnitude. In this manner, the ANPID achieves further customization of control behavior at different error magnitudes via an auto-adjustable gain.

Finally, the discrete time control action $u(n)$ of the ANPID algorithm is being formulated as shown below:

$$
\begin{aligned}
u(n)= & K_{P}\left\{e_{P}(n)+\delta \frac{T_{s}}{T_{I}} \sum_{i=1}^{n}\left[\frac{e_{I}(i)+e_{I}(i-1)}{2}\right] h(i)\right. \\
& \left.+\frac{T_{D}}{T_{s}}\left[e_{D}(n)-e_{D}(n-1)\right]\right\} .
\end{aligned}
$$

where $h(n)=\left(\frac{x_{\text {ref,range }}^{2}}{x_{\text {ref, range }}^{2}+10 e_{I}^{2}(n)}\right)$ defines the discrete representation utilized for the nonlinear adjustment of the internal term in order to counteract overshoot phenomena. In addition, a switch function $\delta$ that is defined in (4):

$\delta=\left\{\begin{array}{ll}1 & \text { for } u_{\min } \leq u(n) \leq u_{\max } \\ 0 & \text { if } u(n) \leq u_{\min } \text { or } u(n) \geq u_{\max }\end{array}\right.$, is incorporated in the integral control action of (3) to avoid the intense overshoot phenomena that follow cases of a constant error factor, due to e.g. a proportional pressure regulator reaching its output limits, where the integral action drives the control effort to its extreme values $u_{\min }$ or $u_{\max }$, resulting in a saturated condition called "wind-up".

In this case, two ANPID algorithms are utilized for the control of the two angular motions, where the manipulated variable $u$ responds to the pressure $\Delta P_{i}$ presented in (1), while the mode selectors are properly defined as $f_{i}, q_{i}$, and $g_{i}$. The process value $x$ is the angular motion $\theta_{i}$ that leads to the following error definition:

$e_{i}=\theta_{i, \text { ref }}-\theta_{i}$

or equivalently denoting the dorsiflexion/plantar flexion and inversion/eversion movement types, defined by $\theta_{1}$ $\left(^{\circ}\right)$ and $\theta_{2}\left(^{\circ}\right)$ respectively, while $\theta_{1}$, ref and $\theta_{2}$, ref are their respective reference angle values. The selected orientation for both process angle values is graphically defined in Fig. 5 and kinetically translated as:

$$
\begin{aligned}
& \theta_{1}\left\{\begin{array}{c}
>0 \Rightarrow \text { Dorsiflexion } \\
<0 \Rightarrow \text { Plantar Flexion }
\end{array}\right. \text { and } \\
& \theta_{2}\left\{\begin{array}{l}
>0 \Rightarrow \text { Eversion } \\
<0 \Rightarrow \text { Inversion }
\end{array}\right.
\end{aligned}
$$

The utilization of PMAs introduces intense nonlinearities, which increase the difficulty in fine-tuning the control parameters $K_{P}, T_{I}$ and $T_{D}$ for achieving efficient control performance throughout the HURL's 
operating range. For this reason, a gain scheduler is appropriately incorporated in the PID control structure, which has the ability to alter the control parameters $K_{P}, T_{I}$ and $T_{D}$ according to the movement type denoted by the switching signal $i$ and according to the active region of operation, specified by the selected manipulated value. In this case, an additional switching signal $m \varepsilon \mathbb{Z}^{+}$is introduced that rules the switching values of the previously constant gains, as in the sequel:

$$
\left[\begin{array}{c}
K_{P}^{i} \\
T_{I}^{i} \\
T_{D}^{i}
\end{array}\right]=\left[\begin{array}{c}
K_{P, m}^{i} \\
T_{I, m}^{i} \\
T_{D, m}^{i}
\end{array}\right] \text { for } m=1,2, \ldots, M,
$$

where $M \varepsilon \mathbb{Z}^{+}$is the maximum number of operating regions. In the utilized notation the sub-indexes $i$ and $m$ are utilized as the switching natures of the corresponding variables.

To ensure smooth transition between the areas of operation defined by the aforementioned gain scheduling mechanism, a bump-less mechanism is incorporated, which acts as an integral sum adjustment function by keeping the addition of the proportional and integral actions invariant to parameter alterations [1]. Therefore, during switches between neighboring areas of operation the control effort becomes:

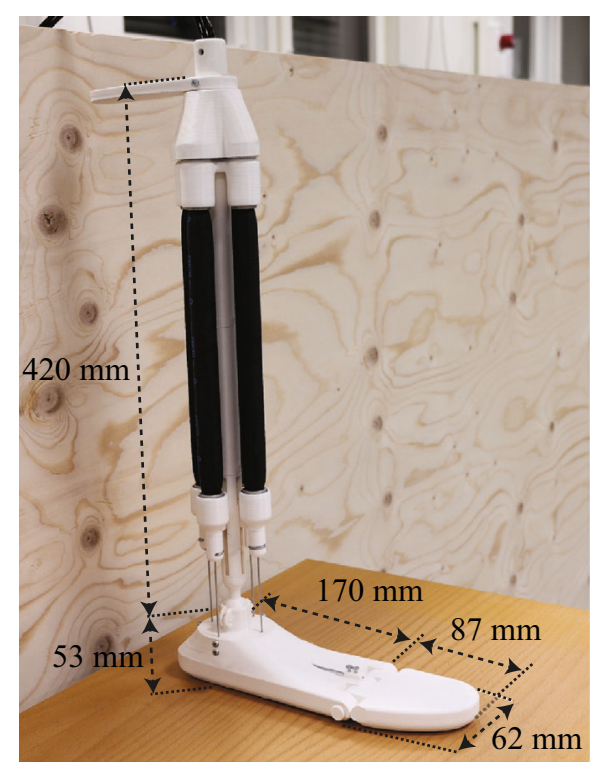

(a)

$$
\begin{aligned}
\Delta P_{i}(n)= & K_{P}^{i}(n-1) e_{P}^{i}(n-1)+K_{P}^{i}(n) \\
& \times\left\{\delta \frac{T_{s}{ }^{n-1}}{T_{I}^{i}} \sum_{i=1}^{i}\left[\frac{e_{I}^{i}(i)+e_{I}^{i}(i-1)}{2}\right] h(i-1)\right. \\
& \left.+\frac{T_{D}^{i}}{T_{s}}\left[e_{D}^{i}(n)-e_{D}^{i}(n-1)\right]\right\}
\end{aligned}
$$

Based on the system's characteristics and the desirable control attributes, constraints are posed on the controllers' outputs $P i, j(n)$ and set-point values $\theta_{i}$, ref $(n)$, by setting minimum and maximum limits as specified in (9):

$$
\left[\begin{array}{c}
P_{i, j}^{\min } \\
\theta_{i, \text { ref }}^{\text {min }}
\end{array}\right] \leq\left[\begin{array}{c}
P_{i, j} \\
\theta_{i, \text { ref }}
\end{array}\right] \leq\left[\begin{array}{c}
P_{i, j}^{\max } \\
\theta_{i, \text { ref }}^{\max }
\end{array}\right]
$$

\section{Prototype development and setup components}

\subsection{Development of the HURL prototype}

For performing a feasibility study on the proposed design, a 2-DoF humanoid leg prototype was developed by following the mechanical strategies described in Sect. 3. The prototype version of the HURL is presented in Fig. 7a, along with highlights of the basic dimensions of its skeletal structure, which follow a

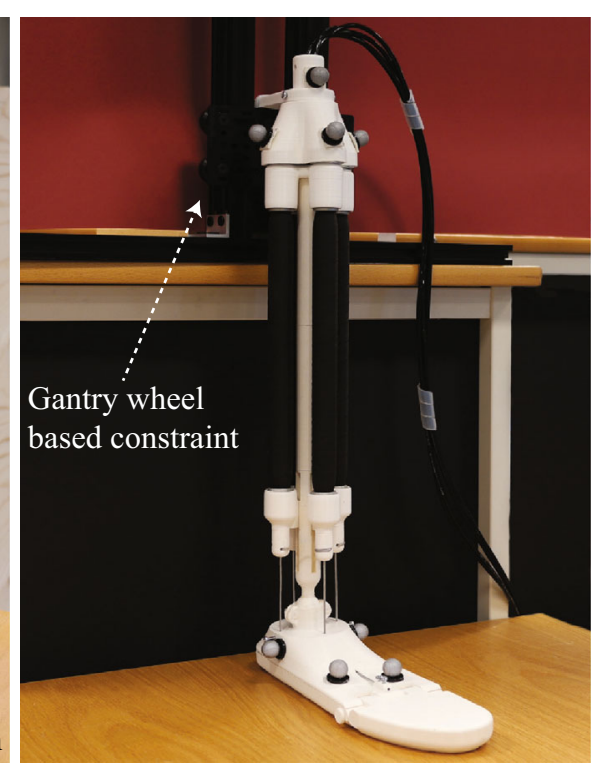

(b)

Fig. 7 The HURL prototype's a dimensions, b evaluation setup 
biomimetic replication of the mean dimensions of an adult male's leg [22]. In addition, Fig. 7b presents the utilized evaluation setup, which had the HURL's knee formation constrained via the use of a gantry wheelbased system so as to allow only its translational movement along the vertical $z$ axis. The purpose of this particular setup was to evaluate the motions of the HURL's ankle while being under load and mechanically forced to replicate the motion patterns displayed in Fig. 5. It has to be noted that the wheel-based setup is passive in nature and does not provide any active motion support on the HURL.

Overall, four Festo DMSP-10-260N-AM-CM Fluidic Muscles with $10 \mathrm{~mm}$ internal nominal diameter and $260 \mathrm{~mm}$ nominal length were utilized for the replication of the ankle movements. The nominal length of the PMAs was appropriately selected by taking into consideration the dimension restrictions of the biomimetic design, while the PMAs' placement upon the skeletal structure of the HURL plays a crucial role to the robot's motion quality, since it acts as a trade-off between the acquired angular motion and the torque generated on the ankle joint, while considering the PMAs' permissible pressure range of 0-8 bar.

In addition, low-weight materials were utilized in the development of the skeletal structure to minimize the inertial effects of the HURL's equipment during joint motion. As shown in Fig. 7, the skeleton, the various connection components and the joint parts have being 3D printed via PLA material. Also, steel wire was selected for the role of the artificial tendons, while being equipped with plastic coating to decrease the friction phenomena during contact with the 3Dprinted joints. This selection of materials led to a robotic leg that weighs approximately $1 \mathrm{~kg}$, of which $0.36 \mathrm{~kg}$ belong to the utilized PMAs.

\subsection{Setup components}

Regarding additional setup components, four Festo VPPM-6F-L-1-F-0L10H-V1N-S1 proportional pressure regulators were utilized to control and measure the pressure of the compressed air supplied into the PMAs. The utilized pressure sensors, which are integrated inside the aforementioned pressure regulators, provide a measurement accuracy of \pm 0.0035 bar. Moreover, a VICON motion capturing system consisted of twenty IR cameras were utilized for the measurement and acquisition of angular and translational motion data of the humanoid leg prototype. The specific equipment ensures high-accuracy measurements, as it provides translation accuracy of approximately $0.04 \mathrm{~mm}$ and a respective angular accuracy of $0.02^{\circ}$. Finally, the control of the setup's operation, as well as the data acquisition, were achieved via a USB$1608 \mathrm{G}$ and a USB-3100 supplied by Measurement Computing, while the setup's programming software was developed in National Instruments LabVIEW.

\section{Experimental results}

Extensive experimental studies were performed to test the HURL's 2-DoFs, as well as to evaluate its performance in reproducing human-like movements. This Section presents the experimentally acquired results, which include open-loop responses for the evaluation of the HURL's structural capabilities in replicating the human ankle's movements, as well as closed-loop control performances via the proposed ANPID-based structure.

At this point it should be highlighted that the main purpose of this article is the design and the implementation of the HURL prototype, as well as the motion control evaluation during this robotic setup's operation, which act as a feasibility study to the future development of BAHRT. The presented research concerns the evaluation of the prototype appliance in ankle-generated angular motions, while the knee is being constrained to a translational movement along its vertical axis. Alternative modes of operation under variable loading, as well as compliance and postural control are future work.

For all the experimental trials described in the sequel, $T_{\mathrm{s}}=0.1 \mathrm{~s}$ was selected as a trade-off between data representation accuracy and overall execution speed based on the utilized computer unit. Moreover, the initial pressures utilized for the joint's idle state were properly tuned as $P_{i,} \quad 0=4 \mathrm{bar}$, while all pressure signals, which were calculated via (1) and the appropriate change of the pressure element $\Delta P_{i, j}$, were constrained to the PMA's permissible range $0 \leq P_{i, j} \leq 8$ bar for $i=1,2$ and $j=1,2$.

Initially, extensive experimental trials concerning open-loop responses were performed in order to test all possible movement patterns of the HURL. Figure 8 presents photographs of the setup during different 


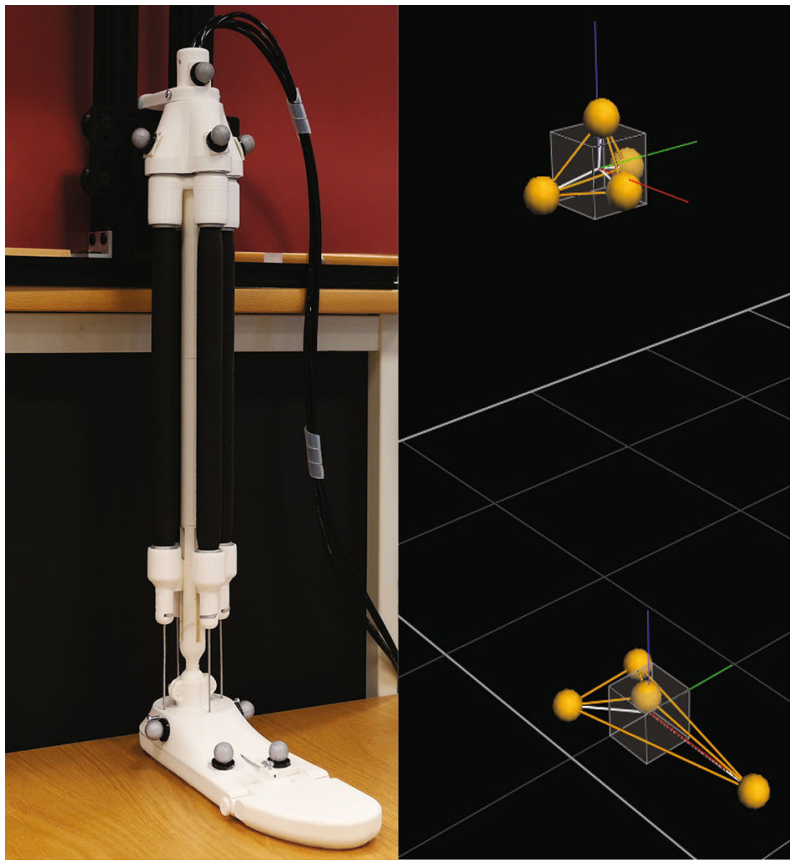

(a)

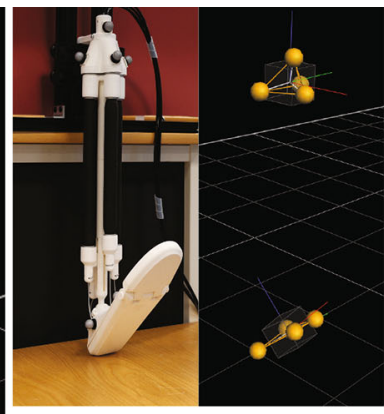

(b)

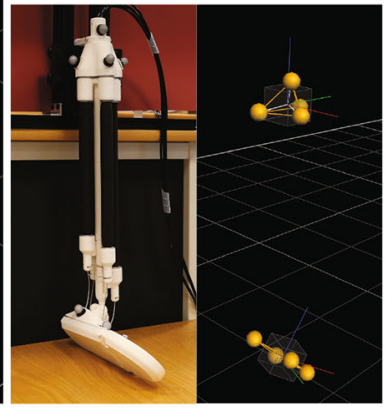

(d)

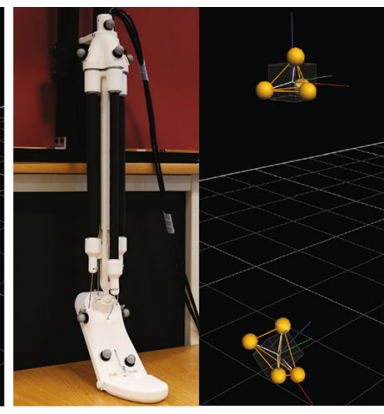

(c)

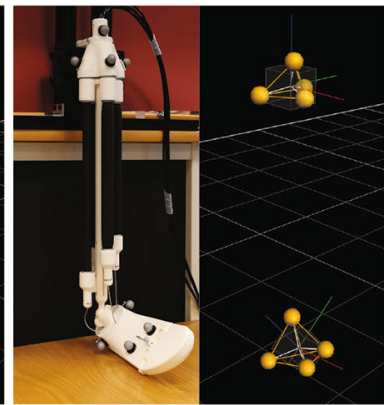

(e)

Fig. 8 Photographic stills and images acquired via the VICON motion capturing system of the HURL prototype during: a idle, b dorsiflexion, c plantar flexion, $\mathbf{d}$ eversion and e inversion

operational states, which were taken throughout these experimental sequences. The angular data of the humanoid ankle were recorded via the VICON motion capturing system, as graphically presented in Fig. 8, thus providing a highly accurate measurement of the maximum angular range of the HURL's joint with respect to the idle state depicted in Fig. 8a and given the maximum pressure range provided via (1).

These maximum motion ranges are presented in Table 1 in comparison to the respective normal ranges of the human foot [23], which show that the HURL possesses the structural capability of replicating the human-like ankle movements displayed in Fig. 5,

Table 1 Range of motion of the HURL prototype

\begin{tabular}{llll}
\hline$i$ & $\begin{array}{l}\text { Ankle } \\
\text { movement }\end{array}$ & \multicolumn{2}{l}{ Maximum angle $\left(^{\circ}\right)$} \\
\cline { 3 - 4 } & HURL & Human foot \\
\hline 1 & Dorsiflexion & 41.2 & $\sim 20$ \\
& Plantar flexion & 39.5 & $\sim 40$ \\
2 & Eversion & 39.0 & $\sim 25$ \\
& Inversion & 39.3 & $\sim 35$ \\
\hline
\end{tabular}

while covering, and in most cases exceeding, the normal range of the human ankle.

Following the open-loop experimental evaluation, the proposed ANPID-based structure undertakes the angular control of multiple dorsiflexion/plantar flexion an eversion/inversion experimental sequences, with the constraints posed on the respective process values $\theta_{1}$ and $\theta_{2}$ specified as $\left[\theta_{1}, \min , \theta_{1}, \max \right]=[-39.4$, $41.3]^{\circ}$ and $\left[\theta_{2, \min }, \theta_{2, \max }\right]=[-39.3,39.1]^{\circ}$. For these experiments, the ANPID mode selectors were empirically adjusted at $f=0.75$ and $q=0$, leading to a linearly adjustable proportional action for balanced disturbance rejection and set-point tracking, along with a measurement-affected derivative action for reduced derivative kick effect in cases of sudden setpoint alterations. The linearity factor $g$ was set to $g=0.81$, in order to achieve a smooth non-linear adjustment of the error signal and a decrease of the $K_{p}$ gain at low error magnitudes. Finally, the gain scheduler's control parameters $K_{P, m}, T_{I, m}$ and $T_{D, m}$ were tuned via fine-tuning through extensive experimental trials and their values, with respect to the PMA's selected operating regions, are presented in Table 2. 
Table 2 ANPID parameters for the HURL's (A) dorsiflexionplantar flexion and (B) eversion-inversion movements

\begin{tabular}{cllll}
\hline$m$ & Operating regions $\left(^{\circ}\right)$ & $K_{\mathrm{P}, m}$ & $T_{\mathrm{I}, m}(\min )$ & $T_{\mathrm{D}, m}(\mathrm{~min})$ \\
\hline (A) & & & & \\
1 & $\theta_{1} \in(-39.4,-20]$ & 0.038 & 0.0021 & 0.0001 \\
2 & $\theta_{1} \in(-30,-20]$ & 0.04 & 0.0020 & 0.0001 \\
3 & $\theta_{1} \in(-20,-10]$ & 0.045 & 0.0020 & 0.0001 \\
4 & $\theta_{1} \in(-10,0]$ & 0.055 & 0.0019 & 0.0002 \\
5 & $\theta_{1} \in(0,10]$ & 0.056 & 0.0023 & 0.0001 \\
6 & $\theta_{1} \in(10,20]$ & 0.049 & 0.0021 & 0.0001 \\
7 & $\theta_{1} \in(20,30]$ & 0.048 & 0.0020 & 0.0002 \\
8 & $\theta_{1} \in(30,41.3]$ & 0.047 & 0.0019 & 0.0003 \\
$(\mathrm{~B})$ & & & & \\
1 & $\theta_{2} \in(-39.3,-30]$ & 0.052 & 0.0021 & 0.0003 \\
2 & $\theta_{2} \in(-30,-20]$ & 0.052 & 0.0023 & 0.0003 \\
3 & $\theta_{2} \in(-20,-10]$ & 0.054 & 0.0020 & 0.0002 \\
4 & $\theta_{2} \in(-10,0]$ & 0.058 & 0.0019 & 0.0002 \\
5 & $\theta_{2} \in(0,10]$ & 0.060 & 0.0020 & 0.0003 \\
6 & $\theta_{2} \in(10,20]$ & 0.062 & 0.0023 & 0.0001 \\
7 & $\theta_{2} \in(20,30]$ & 0.054 & 0.0028 & 0.0002 \\
8 & $\theta_{2} \in(30,39.1]$ & 0.052 & 0.0030 & 0.0003 \\
\hline & & & &
\end{tabular}

Multiple set-point experiments were performed to test the efficacy of the ANPID-based control scheme regarding the HURL's movements. In these trials every DoF is tested separately, while the other is kept at idle state. The experimentally acquired responses are presented in Figs. 9 and 10, respectively, where the multiple reference signals are indicated with red dotted lines and the experimental angular $\theta_{1}$ and $\theta_{2}$ responses are displayed with blue solid lines. For comparison purposes, the experimental data presented in the aforementioned figures are being extended by responses acquired via conventional PID control, denoted as $\theta_{\text {PID }}$ and highlighted by dashed green lines. The conventional three-term strategy excludes the incorporation of a gain scheduling mechanism and utilizes fine-tuned constant gains.

The obtained experimental responses reveal smooth and fast convergence for all test-cases of the ANPID scheme with absence of major transient phenomena. With rising times remaining under half of a second and the mean absolute steady-state errors ranging between $0.02^{\circ}$ and $0.10^{\circ}$ for the dorsiflexion/plantar flexion and $0.03^{\circ}-0.11^{\circ}$ for the eversion/ inversion movements, these set-point experiments indicate the setup's capability to accurately replicate the motion capabilities of the human ankle via the utilization of the proposed control structure.

The need for modifying the conventional algorithm in order to achieve advanced performance best suited for a PMA-actuated device is supported by the respective results acquired via the use of the classic PID structure with constant gains, which are characterized by more intense transient phenomena, larger rising times and steady-state errors ranging between $0.15^{\circ}$ and $0.5^{\circ}$.

In the sequel, experimental studies were performed with the HURL freely suspended for the preliminary evaluation of the system's ability to track both movement patterns at the same time. Specifically, angular responses of $\theta_{1}$ and $\theta_{2}$ for a sinusoidal reference signal ranging between $-30^{\circ}$ and $30^{\circ}$ at $0.2 \mathrm{~Hz}$, while being characterized by a $90^{\circ}$ phase difference, are depicted in Fig. 11, along with their respective error signals $e_{i}=\theta_{i}$, ref $-\theta_{i}$ and pressure control efforts $P_{i, j}$. The performance quality of the ANPID control structure is maintained, providing smooth tracking in both cases despite the selected angular reference input covers a large part of the HURL's operational range, as well as the frequency of the reference signals by taking into account the inherently large settling times of the utilized PMAs. The mean absolute error of the proposed scheme remains at low values and is measured at $0.89^{\circ}$ for the dorsiflexion/plantar flexion and $0.84^{\circ}$ for the eversion/ inversion movements.

Finally, experimental responses via sinusoidal reference signals of the same aforementioned attributes but frequencies increasing from 0.2 to $0.6 \mathrm{~Hz}$ are presented in Fig. 12. The control structure manages to successfully control the two angular motions of the HURL in all test-cases, although as expected, the increase in frequency reveals a progressive increase in the mean absolute errors, which at $0.6 \mathrm{~Hz}$ reach $1.84^{\circ}$ for the dorsiflexion/plantar flexion and $1.83^{\circ}$ for the eversion/inversion movements. Nonetheless, these control performances are considered sufficient given the challenging reference trajectories and further highlight the HURL's mechanical ability in reproducing the ankle movements in an accurate and smooth manner.

The maximum frequency selection for the aforementioned responses is ultimately governed by the bandwidth of the system at the specific reference states. With decreased angular ranges the utilized 
Fig. 9 Set-point experiments for the evaluation of the HURL's dorsiflexion and plantar flexion movement capabilities
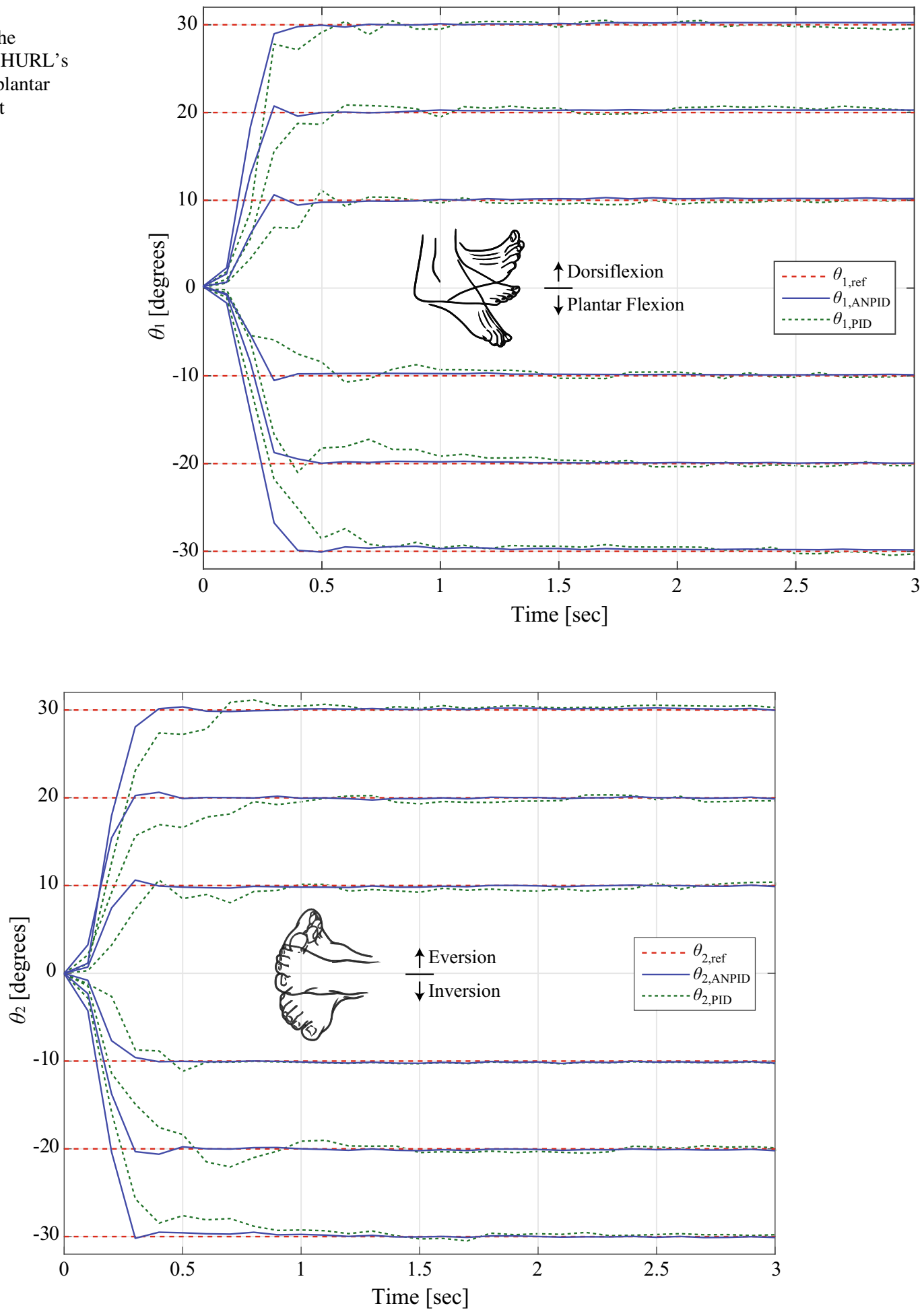

Fig. 10 Set-point experiments for the evaluation of the HURL's eversion and inversion movement capabilities 
Fig. 11 Set-point experiments for the evaluation of the HURL's dorsiflexion and plantar flexion movement capabilities

Fig. 12 Set-point experiments for the evaluation of the HURL's eversion and inversion movement capabilities

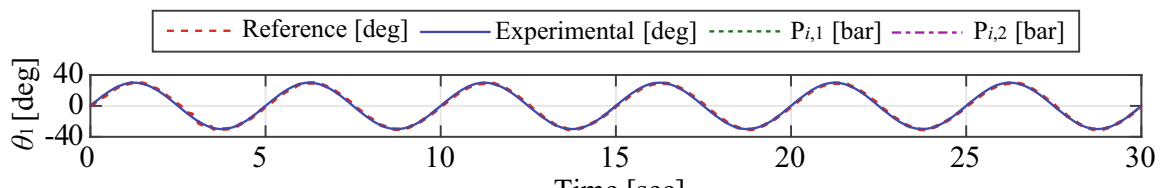

Time [sec]

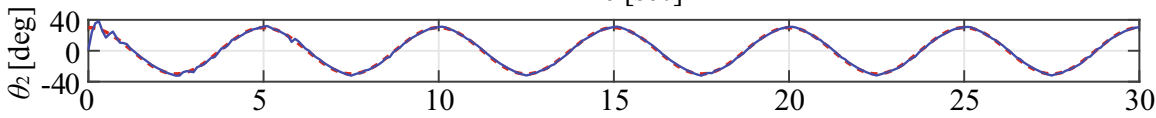

Time [sec]
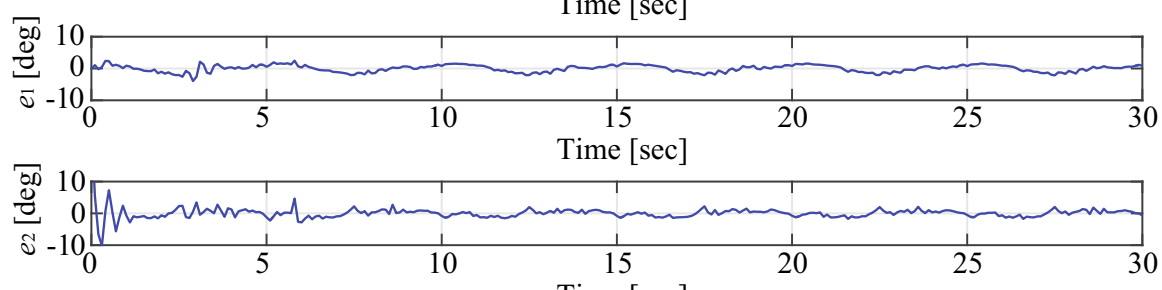

Time [sec]

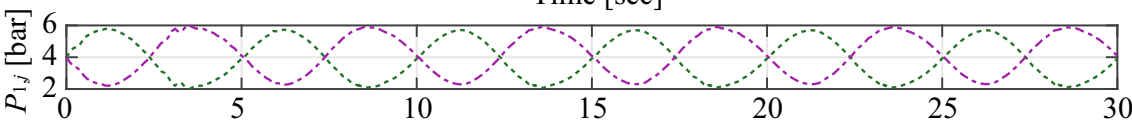

Time [sec]

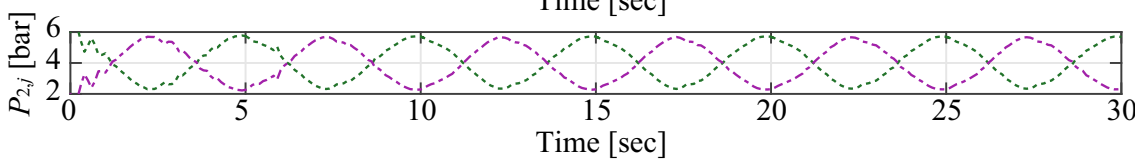

Time [sec]
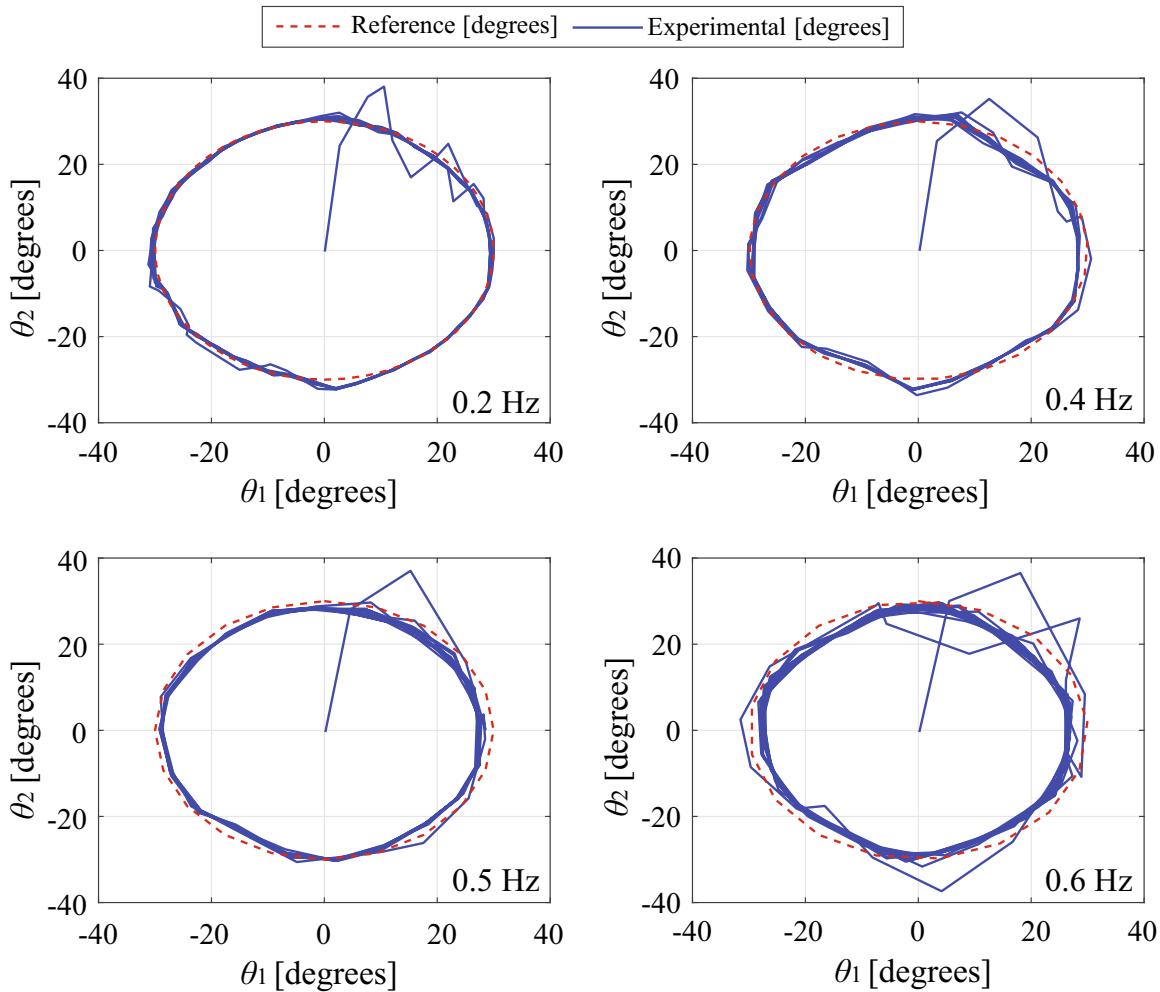
PMAs are able to reach higher frequencies of up to $5 \mathrm{~Hz}$, which corresponds to tremor-based motion patterns. The evaluation of the HURL performance in such challenging scenarios and with the integration of different control strategies [24] is considered future work.

It has to be highlighted that the utilized robust control scheme was selected for the preliminary evaluation of the prototype and does not provide an optimal solution in the strict theoretical sense of the term. The presented responses were acquired after a series of experimental trials performed for the finetuning of the ANPID mode selectors and gains, while the incorporation and testing of dynamic model-based optimal schemes is part of future work.

The experimental results presented in this Section prove the conceptual assumption of a design where its 2 DoFs are mechanically decoupled and can be successfully controlled via two independent controllers, thus highlighting the design's advantage of simplifying the complexity of this demanding motion control problem. In overall, the experimental evaluation reveals that this novel endoskeletal design approach possesses the structural attributes necessary for biomimetic reproduction of the basic movements of the human ankle and enables the expansion of this design the proposed 10-DoF lower-limb configuration.

\section{Conclusions}

In this article, the design and development problem of a HUmanoid Robotic Leg (HURL) prototype was addressed. The proposed structure concerned the conceptualization of a biomimetic design for mechanically decoupled actuation of both DOFs of an ankleinspired joint. To this purpose, the synergistic nature of the human lower leg muscles was simplified to two antagonistic pairs of PMAs in cross-formation, thus providing the structural ability of independent control of motions in the sagittal and frontal plane. The proposed approach utilized a 3D-print-friendly design, which allowed for fast, low-cost prototyping and easy customization of its sub-parts to given dimension requirements. For the evaluation of the conceptual design strategy, a feasibility study was performed via the development of a 2-DoF prototype and the results of a preliminary experimental evaluation of its motion capabilities (extension-flexion, ulnar-radial deviation) were presented. Multiple tracking sequences were performed via an advanced non-linear PID-based control algorithm and the acquired results proved that the HURL possesses the structural attributes necessary for biomimetic reproduction of the basic movements of the human ankle in a smooth and accurate manner.

\section{Future work}

The authors have to note that the presented research concerned the preliminary evaluation of the HURL prototype, where the 2-DoF structure was considered as a basic motion problem. However, the conceptual design from an engineering point of view and without losing generalization forms the basis for its expansion in developing a compliant, safe and motion accurate lower-body humanoid for future research in balance and postural control.

Considering the goal of this article, which was to present the conceptual design and a preliminary experimental evaluation of its structural capabilities via a model-free control structure, the important matters of dynamic modeling and stability analysis are considered matters of future work. Alternative model-based control structures for postural control will also be considered and evaluated for various balance strategies, where the HURL's efficiency will be tested both as a prosthetic limb, as well as a part of a biped robotic setup.

Acknowledgements This study was funded by Swedish Research Council (Vetenskapsrådet) (Grant No. 2014-3381).

\section{Compliance with ethical standards}

Conflict of interest The authors declare that they have no conflict of interest.

Open Access This article is distributed under the terms of the Creative Commons Attribution 4.0 International License (http:// creativecommons.org/licenses/by/4.0/), which permits unrestricted use, distribution, and reproduction in any medium, provided you give appropriate credit to the original author(s) and the source, provide a link to the Creative Commons license, and indicate if changes were made.

\section{References}

1. Bekey GA (2008) Robotics: state of the art and future challanges. Imperial College Press, London 
2. Wang J (2008) A survey on the structures of current mobile humanoid robots. In: Proceedings of IEEE Asia Pacific Conference on Circuits and Systems (APCCAS), 30 November-3 December 2008, Macao

3. Al-Shuka HFN, Allmendinger F, Corves B, Zhu W-H (2014) Modeling, stability and walking pattern generators of biped robots: a review. Robotica 32(6):907-934

4. Andrikopoulos G, Nikolakopoulos G, Manesis S (2013) Adaptive internal model control scheme for a pneumatic artificial muscle. In: European Control Conference (ECC), 17-19 July 2013, Zurich, pp 772-777

5. Andrikopoulos G, Nikolakopoulos G, Manesis S (2015) Design and development of an exoskeletal wrist prototype via pneumatic artificial muscles. In: Meccanica, special issue on soft mechatronics: mechanics and multi-physics of compliant transducers, vol 50, no 11. Springer, pp 2709-2730, November 2015

6. Colbrunn RW, Nelson GM, Quinn RD (2001) Design and control of a robotic leg with braided pneumatic actuators. In: IEEE/RSJ international conference on intelligent robots and systems, vol 2, pp 992-998

7. Verrelst B, Van Ham R, Vanderborght B, Daerden F, Lefeber D, Vermeulen J (2005) The pneumatic biped 'lucy' actuated with pleated pneumatic artificial muscles. Auton Robots 18(2):201-213

8. Versluys R, Naudet J, Vanderborght B, Lenaerts G, Lefeber D (2006) IPPAM: Intelligent prosthesis actuated by pleated pneumatic artificial muscles: objectives and mechanical concept. In: International Conference on Climbing and Walking Robots (CLAWAR), pp 1-9

9. Takuma T, Hayashi S, Hosoda K (2008) 3D bipedal robot with tunable leg compliance mechanism for multi-modal locomotion. 2008 IEEE/RSJ International Conference on Intelligent Robots and Systems, pp 1097-1102, September 2008

10. Tahboub KA (2009) Biologically-inspired humanoid postural control. J Physiol Paris 103(3-5):195-210

11. Narioka K, Tsugawa S, Hosoda K (2009) 3D limit cycle walking of musculoskeletal humanoid robot with flat feet. 2009 IEEE/RSJ International Conference on Intelligent Robots and Systems, pp. 4676-4681, Oct. 2009

12. Niiyama R, Nishikawa S, Kuniyoshi Y (2010) Athlete robot with applied human muscle activation patterns for bipedal running. In: 10th IEEE-RAS International Conference on Humanoid Robots, pp 498-503

13. Lei J, Wu J, Yu H (2014) Analysis on the musculoskeletal leg mechanism driven by PMAs. In: 2014 IEEE International Conference on Mechatronics and Automation, pp 1659-1663

14. Bierbaum A, Schill J, Asfour T, Dillmann R (2009) Force position control for a pneumatic anthropomorphic hand. In: IEEE/RAS International Conference on Humanoid Robots (Humanoids), pp 21-27

15. Jiang F, Tao G, Liu H (2015) Research on PMA properties and humanoid lower limb application. In: IEEE/ASME International Conference on Advanced Intelligent Mechatronics (AIM), Busan, pp 1292-1297

16. Jiang F, Tao G, Li Q (2017) Analysis and control of a parallel lower limb based on pneumatic artificial muscles. Adv Mech Eng 9(1):1-14

17. Drake R (2015) Gray's anatomy for students, 3rd edn. Elsevier, Amsterdam

18. Andrikopoulos G, Nikolakopoulos G, Manesis S (2014) Advanced non-linear PID based antagonistic control for pneumatic muscle actuators. IEEE Trans Ind Electron 61(12):6926-6937

19. Tondu B, Lopez P (2000) Modeling and control of McKibben artificial muscle robot actuators. IEEE Control Syst Mag 20(2):15-38

20. Netter FH (2014) Atlas of human anatomy, 6th edn. W. B. Saunders Company, Philadeiphia

21. Åström KJ, Hagglund T (1995) PID controllers: theory, design and tuning. Instrument Society of America, Research Triangle Park, NC, pp 343-345

22. Winter D (2009) Biomechanics and motor control of human movement, 4th edn. Wiley, Hoboken

23. Nigg BM, Fisher V, Allinger TL, Ronsky JR, Engsberg JR (1992) Range of motion of the foot as a function of age. Foot Ankle 13(6):336-343

24. Andrikopoulos G, Nikolakopoulos G, Arvanitakis I, Manesis S (2013) Switching Model Predictive Control of a Pneumatic Artificial Muscle. In: International Journal of Control, Automation, and Systems (IJCAS), vol 11, no 6. Springer, December 2013 